\title{
Ablation laser d'oxydes : étude du transfert de l'oxygène lors de la synthèse de films minces
}

\author{
E. Millon
}

\author{
LSMCL, Institut de Physique Électronique et de Chimie, Université de Metz, 1 boulevard Arago, \\ Technopôle Metz 2000, 57078 Metz cedex 3, France
}

\begin{abstract}
Résumé : Les mécanismes liés au transport de l'oxygène entre la cible et le substrat au cours de la préparation de couches minces d'oxydes par la technique Pulsed Laser Deposition (PLD) sont illustrés ici par le biais d'exemples de travaux sur la réalisation de films de pérovskites $\left(\mathrm{PbTiO}_{3}, \mathrm{PbZr}_{\mathbf{x}} \mathrm{Ti}_{1-\mathrm{x}} \mathrm{O}_{3}\right)$ et de cuprates supraconducteurs à haute température critique ("YBaCuO" et "BiSrCaCuO"). Les différents modes d'incorporation de l'oxygène dans les couches minces ainsi que son rôle dans le transfert des espèces en phase gazeuse entre la cible et le substrat sont discutés. La présence de l'oxygène introduit dans l'enceinte d'ablation au cours de la PLD et lors du refroidissement influence les processus d'oxydation statique de la couche en croissance et conditionne la stabilité relative des espèces oxygénées (ions, molécules, agrégats) dans le plasma. Ce dernier aspect permet en particulier de contrôler le transfert de stoechiométrie des éléments les plus volatils ( $\mathrm{Pb}, \mathrm{Li}$...).
\end{abstract}

\section{INTRODUCTION}

Depuis plus d'une décennie, la technique de dépôt par ablation laser ou PLD (Pulsed Laser Deposition) a montré tout son intérêt pour la croissance épitaxiale de films minces de céramiques supraconductrices à haute température critique. Depuis, elle a été appliquée avec succès à la synthèse d'autres matériaux qu'ils soient de nature métallique, isolante, semi-conductrice et polymérique. L'ablation laser des céramiques (oxydes, nitrures, carbures...) demeure actuellement très étudiée pour la préparation de films minces. En particulier, les oxydes hormis bien évidemment les cuprates, peuvent être obtenus par PLD sous forme de films minces avec de bonnes qualités morphologique et cristalline pour de nombreux domaines d'application : citons en particulier, les pérovskites ferroélectriques (PZT, PLT, $\left.\mathrm{PbTiO}_{3} \ldots\right)$, les conducteurs ioniques $\left(\mathrm{LiMn}_{2} \mathrm{O}_{4}, \mathrm{LiCoO}_{2} \ldots\right)$, et plus récemment les oxydes pour laser et optique non linéaire (YAG dopé, KDP, borates, niobates...).

La qualité des films obtenus dépend des conditions de croissance qui dans le cas des oxydes imposent d'effectuer la PLD en atmosphère réactive d'oxygène sous pression contrôlée (typiquement de $10^{-5}$ à quelques mbar). L'oxygène de confinement et celui apporté par les produits d'ablation de la cible va en premier lieu influencer la stœchiométrie en oxygène du film obtenu mais aussi les conditions de croissance de la couche. Par le biais d'exemples de travaux relatifs principalement aux céramiques supraconductrices à haute température critique et aux pérovskites $\mathrm{ABO}_{3}$, nous illustrerons ici les différents modes d'incorporation de l'oxygène dans les couches minces ainsi que son rôle dans le transfert des espèces en phase gazeuse entre la cible et le substrat.

\section{LES DIFFERENTES VOIES D'INCORPORATION DE L'OXYGENE}

La conservation de la stœchiométrie dans le cas de l'ablation laser de matériaux de composition chimique complexe est une des caractéristiques principales de la PLD qui est d'ailleurs à l'origine de son succès. Néanmoins, ce fait doit être pondéré dans le cas des oxydes pour lesquels il est maintenant bien connu qu'il est nécessaire d'effectuer l'ablation de la cible en présence d'un gaz oxydant pour corriger le déficit en oxygène [1] provenant de processus complexes liés au transport des espèces en phase gazeuse dans le plasma suite à l'interaction du faisceau laser avec la cible.

Le gaz réactif (généralement l'oxygène pour la PLD des oxydes) introduit dans l'enceinte d'ablation permet non seulement de contrôler la stœechiométrie du dépôt par rapport à la teneur en oxygène, mais aussi de favoriser la fixation sur la couche en croissance d'éléments volatils comme le plomb ou le lithium. Par exemple, il est impératif de contrôler la pression d'oxygène ainsi que la température du substrat pour stabiliser la phase pérovskite lors de la croissance de films minces de $\mathrm{PbTiO}_{3}[2,3]$. Fait moins connu, l'oxygène de confinement peut également influer sur la structure et l'orientation cristalline d'une couche mince métallique en favorisant une croissance préférentielle selon des plans 
cristallographiques denses comme en témoignent les travaux récents sur la croissance de films minces de $\mathrm{Pt} / \mathrm{MgO}[4]$.

L'oxygène incorporé dans la couche mince d'oxyde peut être issu de différentes étapes du processus d'ablation laser. On peut ainsi envisager différentes voies d'incorporation de l'oxygène :

- l'une traduit une oxydation dite" statique" qui concerne d'une part la contribution de la phase de refroidissement survenant à la fin du processus d'ablation proprement dit et qui est généralement effectuée sous pression d'oxygène contrôlée. D'autre part, cela concerne l'incorporation de l'oxygène durant l'intervalle de temps entre deux impulsions laser successives;

- l'autre prend en compte des processus d'oxydation dite "dynamique" qui regroupent la part de l'oxygène incorporée par réaction en phase gazeuse des espèces ablatées de la cible. Dans ce cas, on peut distinguer les espèces oxygénées issues directement de la cible de celles résultant de réaction d'échange avec le gaz réactif.

Pour étudier le rôle précis de l'oxygène dans le processus de dépôt, il est possible d'utiliser une technique de marquage isotopique à l'oxygène 18 qui consiste à remplacer l'oxygène ambiant par de l'oxygène fortement enrichie en ${ }^{18} \mathrm{O}_{2}(98 \%)$ pendant l'expérience de PLD. Cette méthode a été utilisée initialement pour le cas des céramiques supraconductrices "YBaCuO" [5] et "BiSrCaCuO" [6] et plus récemment pour la pérovskite $\mathrm{PbTiO}_{3}$ [7]. Le dosage des deux isotopes de l'oxygène ${ }^{16} \mathrm{O}$ et ${ }^{18} \mathrm{O}$ incorporé dans le film mince peut être réalisé par spectrométrie d'ions secondaires (SIMS) en mode dynamique avec des ions primaires $\mathrm{Cs}^{+}$. L'intensité des signaux correspondants aux ions secondaires $\mathrm{Cs}^{16,18} \mathrm{O}^{+}$permet d'obtenir la proportion relative des deux isotopes dans le film [7]. Une autre méthode de dosage des isotopes de l'oxygène dans les couches minces est basée sur la spectroscopie de rétrodiffusion des ions ${ }^{4} \mathrm{He}^{+}$(RBS) et sur l'analyse par réactions nucléaires (NRA) selon les réactions : ${ }^{16} \mathrm{O}(\mathrm{d}, \mathrm{p}){ }^{17} \mathrm{O}$ et ${ }^{18} \mathrm{O}(\mathrm{p}, \alpha){ }^{15} \mathrm{~N}[6]$. Les figures 1 et 2 représentent des exemples d'analyses menées respectivement par RBS sur un film de $\mathrm{Bi}_{2} \mathrm{Sr}_{2} \mathrm{Ca}_{1} \mathrm{Cu}_{2} \mathrm{O}_{8} / \mathrm{MgO}$ et par SIMS dynamique sur une couche

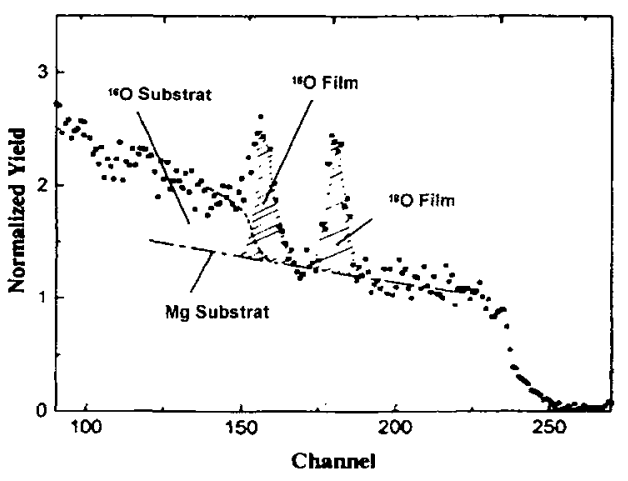

mince $\mathrm{PbTiO}_{3} / \mathrm{Pt}$.

Figure 1 : spectre RBS $\left({ }^{4} \mathrm{He}^{+}, 2,4 \mathrm{MeV}\right)$ en géométrie de canalisation d'un film $\mathrm{BiSrCaCuO} / \mathrm{MgO}$ obtenu à $700^{\circ} \mathrm{C}$ [7].

sous 0,2 mbar ${ }^{18} \mathrm{O}_{2}$ d'après [6].

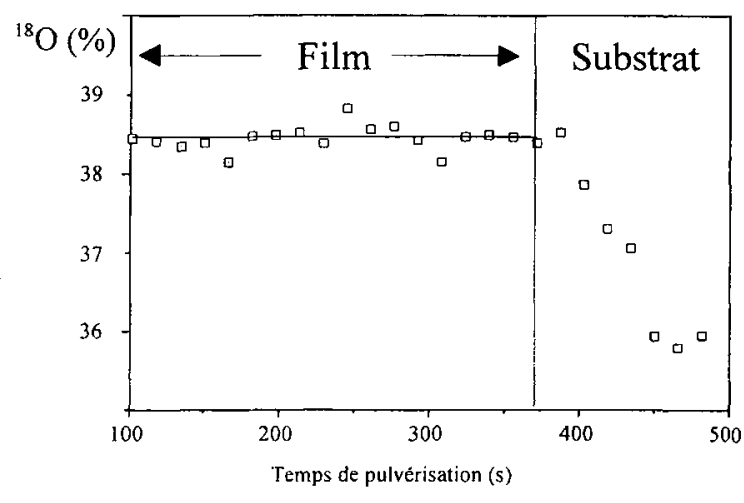

Figure 2 : profil de concentration SIMS en ${ }^{18} \mathrm{O}$ d'un film $\mathrm{PbTiO}_{3} / \mathrm{Pt}$ obtenu à $550^{\circ} \mathrm{C}$ sous 0,3 mbar ${ }^{18} \mathrm{O}_{2}$ d'après

En modulant différents paramètres expérimentaux lors de l'ablation laser (atmosphère de refroidissement, fluence et fréquence de tir du laser) on peut déterminer. la part respective des différentes voies d'incorporation de l'oxygène dans les films. On peut ainsi montrer que $55 \%$ de l'oxygène provient directement de l'ablation de la cible (dans le cas d'une cible d'oxyde). La fraction restante $(45 \%)$ est d'une part, incorporée suite à des réactions de transfert en phase gazeuse avec l'oxygène de confinement (processus dit "dynamique") et d'autre part, introduite par des processus d'oxydation dit "statique" au cours de l'étape de refroidissement après la synthèse par PLD du film. Il convient de remarquer que la pression d'oxygène imposée lors du refroidissement n'a pas la même conséquence au niveau de la quantité d'oxygène introduit selon le type de matériaux synthétisés. Ainsi, les films minces de céramiques supraconductrices préparées par PLD nécessitent des pressions d'oxygène élevées lors du refroidissement (par exemple 500 Torr $\left(6,6.10^{4} \mathrm{~Pa}\right.$ ) pour "BiSrCaCuO") afin d'obtenir une couche mince satisfaisante sur le plan de la supraconductivité. Dans ce cas, l'oxygène présent dans le film provient à $90 \%$ de l'étape de refroidissement [8] alors que sa teneur n'excède pas $45 \%$ lorsque le film est refroidi sous vide. Cette proportion de $45 \%$ est également obtenue pour l'oxyde $\mathrm{PbTiO}_{3}$ pour lequel il est nécessaire d'effectuer le refroidissement sous 0,3 mbar (30 Pa) afin de garder la structure pérovskite [7]. Sans préjuger du mécanisme exact d'incorporation qui survient lors du 
refroidissement à la surface de la couche mince au contact avec l'oxygène, on peut constater que l'incorporation de l'oxygène de confinement est favorisée par la présence de joints de grains et de porosité dans le matériau, et survient de manière prépondérantes lorsque la température est élevée (au début du refroidissement).

L'oxydation statique peut également avoir lieu dans l'intervalle de temps entre deux pulses laser successifs lors de l'étape de PLD proprement dite. Cet aspect est donc en relation directe avec la fréquence de tir du laser employé. A l'heure actuelle, les cadences de tirs habituellement utilisées dans les expériences de PLD avec des lasers de type Nd: YAG (doublé ou quadruplé) ou excimères (ArF ou $\mathrm{KrF}$ ) sont de l'ordre de la dizaine de $\mathrm{Hz}$. Dans cette gamme de fréquence (entre 1 et $10 \mathrm{~Hz}$ ) on ne note pas d'effet marqué sur l'incorporation de l'oxygène dans les couches minces d'oxydes étudiés $[5,6,7]$. Néanmoins, l'emploi de laser à haute cadence de tir (plusieurs centaines de $\mathrm{Hz}$ ) pourrait modifier ces données et induire des modifications de teneur en oxygène dans les films comparativement à des couches minces obtenues avec la même pression d'oxygène dans l'enceinte mais avec une cadence de tir laser plus faible.

Le plasma en expansion est le siège de processus complexes sur le plan thermodynamique et cinétique (distribution spatiale, énergie et vitesse des espèces éjectées) et d'un point de vue physico-chimique (nature et composition des espèces, proportion respective en ions, électrons, espèces neutres et agrégats). Aussi, l'énergie apportée par unité de surface au niveau du matériau cible va influer sur les processus d'ablation et par voie de conséquence sur les réactions se produisant en phase gazeuse. Il a été montré [7] qu'un accroissement de la fluence du laser $(266 \mathrm{~nm})$ entre 1,5 et $3 \mathrm{~J} / \mathrm{cm}^{2}$ lors la synthèse de pérovskite dans les conditions standard $\left(550^{\circ} \mathrm{C}, 0,3 \mathrm{mbar}\right)$ conduisait à une augmentation substantielle (environ $10 \%$ ) de l'incorporation de l'oxygène de confinement dans la couche mince. Cela peut traduire une formation accrue d'oxygène atomique par photodissociation du gaz au voisinage de la cible et par collision avec les espèces du plasma. A fluence élevée, la dissociation de l'oxygène à la surface du film en formation par les espèces ablatées plus énergétiques, peut être également plus conséquente.

\section{LE TRANSPORT DES ESPECES EN PHASE GAZEUSE}

L'oxygène introduit sous forme gazeuse conditionne évidemment la teneur en oxygène dans le film mais aussi permet d'assurer un transfert stœchiométrique d'éléments volatils comme le plomb. Des mécanismes complexes conduisent à une meilleure fixation de ceux ci à la surface du film en croissance, mais aussi, comme nous allons le voir, à une stabilité relative accrue des espèces oxygénées du plomb en phase gazeuse. Dans le cas des pérovskites de type PZT $\left(\mathrm{PbZr}_{\mathrm{x}} \mathrm{Ti}_{1-\mathrm{x}} \mathrm{O}_{3}\right)$ entre autres, il est connu que le couple pression d'oxygène - température du substrat est prépondérant vis à vis de la stœechiométrie en plomb dans le film : pour des températures supérieures à $500^{\circ} \mathrm{C}$ qui sont indispensables à l'obtention d'une phase cristallisée, il est nécessaire de travailler sous 0,3 mbar d'oxygène dans l'enceinte pour avoir la bonne composition en plomb [9].

Au dessus du seuil d'ablation, les impulsions laser induisent la formation d'un plasma lumineux qui se développe perpendiculairement à la surface de la cible. Lorsque l'ablation est menée sous vide ou sous gaz inerte (argon), le plasma prend une coloration blanche alors qu'il devient bleu en présence d'oxygène. Cette coloration de la plume en présence d'oxygène résulte de l'émission d'espèces excitées présentes en phase gazeuse, phénomène induit par des effets collisionnels entre les molécules du gaz dans l'enceinte et les espèces ablatées.

La caractérisation de la plume d'ablation sur le plan physique (distributions cinétique et spatiale des espèces présentes, température, densité électronique..) et chimique (nature et proportion des différentes espèces : ions élémentaires, atomes et molécules excités, agrégats moléculaires ou ionisés) requiert des techniques analytiques basées sur la spectrométrie d'émission optique et la spectrométrie de masse. La première qui peut être résolue dans le temps et dans l'espace [10], permet d'accéder à des informations in-situ concernant les espèces neutres ou excitées (qui sont les espèces prépondérantes dans les plasmas de PLD) mono- ou diatomique avec une limitation due à la difficulté d'interprétation des spectres d'émission. La spectrométrie de masse quant à elle, s'avère complémentaire par sa capacité à caractériser les ions monoatomiques jusqu'aux agrégats ionisés (édifices polyatomiques chargés positivement ou négativement) de haute masse $(\mathrm{m} / \mathrm{z}>1000)[11]$.

Les perovskites ont fait l'objet d'un certain nombre de travaux de caractérisation de plumes d'ablation qui permettent de mieux comprendre le rôle de l'oxygène ambiant sur la stabilité relative des espèces en phase gazeuse. Quelques exemples d'études spécifiques vont être décrits ci-après.

Grâce à une caméra CCD munie de filtres sélectifs en longueur d'onde, Chen et al. [12] ont montré que le plasma d'ablation d'une cible de $\mathrm{PbTiO}_{3}$ par un laser excimère à $248 \mathrm{~nm}$ présentait un comportement singulier sous $\mathrm{O}_{2}$ comparativement aux ablations menées sous $\mathrm{N}_{2}$ ou Ar. Sous $\mathrm{O}_{2}$ uniquement, la présence d'une zone du plasma émissive à des longueurs d'onde supérieures à $600 \mathrm{~nm}$ est caractéristique de l'émission d'atomes d'oxygène, de molécules $\mathrm{O}_{2}$ et d'entité moléculaire correspondant 
au dimère TiO. Le domaine d'émission correspondant à des longueurs d'onde de 400 à $500 \mathrm{~nm}$ est similaire sous les trois atmosphères d'ablation et il correspond aux espèces atomiques et ionisées du titane et du plomb.

La caméra CCD ne donnant qu'une information globale sur le domaine spectrale d'émission des espèces, il est nécessaire d'utiliser la spectroscopie d'émission pour attribuer les raies d'émission à des espèces précises. En utilisant une fibre optique dont l'extrémité est située à $5 \mathrm{~mm}$ de la cible ablatée et un détecteur muticanal sensible à deux domaines de longueurs d'onde (340-415 nm et 555-630 nm), Barnes et al. [10] ont étudié les plasmas d'ablation par un laser $\mathrm{KrF}(248 \mathrm{~nm})$ de $\mathrm{PbO}, \mathrm{TiO}_{2}, \mathrm{ZrO}_{2}$ et de $\mathrm{Pb}\left(\mathrm{Zr}_{0.52} \mathrm{Ti}_{0.48}\right) \mathrm{O}_{3}$ sous différentes pressions d'oxygène $\left(10^{-3}\right.$ à $\left.900 \mathrm{mTorr}\right)$. A titre d'exemple, la figure 3 montre les spectres d'émission correspondant aux deux domaines précités pour l'ablation de $\mathrm{ZrO}_{2}$ sous $900 \mathrm{mTorr}(120 \mathrm{~Pa}) \mathrm{d}^{\prime} \mathrm{O}_{2}$.
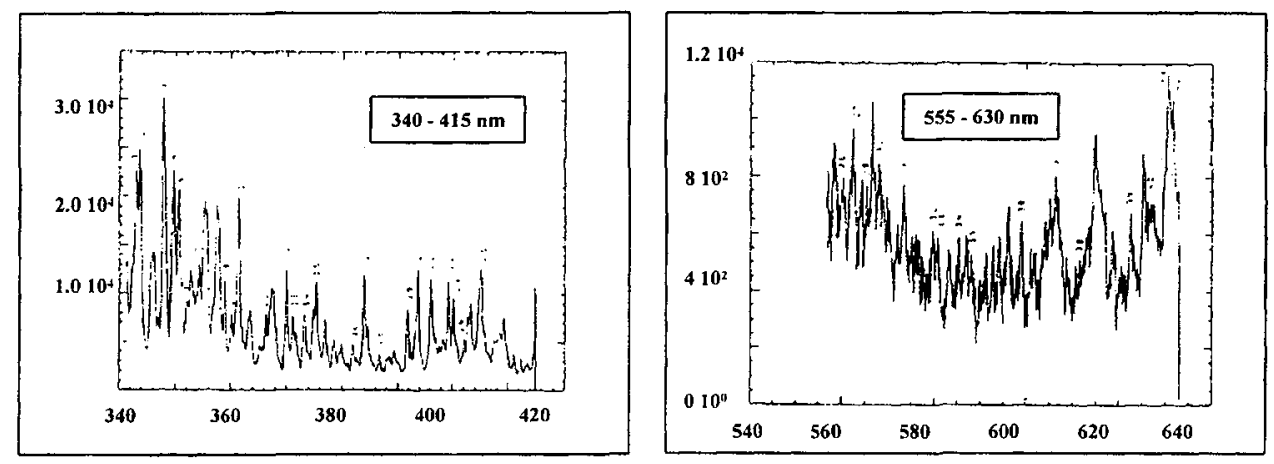

Figure 3 : photoluminescence d'un plasma d'ablation laser à $248 \mathrm{~nm}$ de $\mathrm{ZrO}_{2}$ sous $900 \mathrm{mTorr}$ d'O $\mathrm{O}_{2}$ d'après [10]

Malgré la complexité des spectres liés à un nombre élevé de raies d'émission, les auteurs identifient des espèces telles que des atomes métalliques neutres à l'état excité $M(\mathrm{I})$, des ions excités $M(\mathrm{II})$ chargés +1 ou M(III) chargés +2 ainsi que des dimères neutres ou ionisés du type $\mathrm{MO}_{\mathrm{n}}$ ou $\mathrm{MO}_{\mathrm{n}}{ }^{+}$avec $\mathrm{n}$ égal à 1 ou 2. Le comportement de $\mathrm{PbO}$ est singulier puisque, pour cet oxyde, les espèces de type $\mathrm{PbO}$ ou $\mathrm{PbO}_{2}$ neutres ou chargées ne sont pas détectées alors que l'ablation des autres cibles conduit à la détection des espèces en phase gazeuse $\mathrm{MO}_{\mathrm{n}}$ pour $\mathrm{M}=\mathrm{Ti}$ et $\mathrm{Zr}$. Ce fait peut être relié à la plus faible enthalpie de vaporisation de $\mathrm{PbO}(158 \mathrm{kcal} / \mathrm{mol})$ comparativement à celles de $\mathrm{TiO}_{2}(466 \mathrm{kcal} / \mathrm{mol})$ et de $\mathrm{ZrO}_{2}(524$ $\mathrm{kcal} / \mathrm{mol}$ ) ce qui engendre une instabilité relative des espèces oxygénées à base de plomb dans le plasma. Les auteurs notent également un accroissement de l'abondance relative des espèces $\mathrm{MO}_{\mathrm{n}}$ quand la pression d'oxygène dans l'enceinte augmente. Bien que purement qualitatif, cet effet permet de conclure que l'oxygène ambiant favorise la formation d'espèces oxygénées en phase gazeuse ce qui est compatible avec les conditions d'ablation utilisées en PLD pour obtenir des films de bonne qualité.

En effectuant un diagnostic plasma par spectrométrie de masse à temps de vol, on peut étudier l'abondance relative des agrégats ionisés [13]. En particulier dans le cas de l'ablation du titanate de plomb $\mathrm{PbTiO}_{3}$, les agrégats de haute masse de type $\left[\mathrm{A}(\mathrm{B})_{n}\right]^{+/-}$sont formés par réaction d'addition dans le plasma d'une molécule neutre $B$ sur un ion précurseur $A^{+/-}$. Les espèces neutres sont dans le cas présent majoritairement $\mathrm{TiO}_{2}$ et à un degré moindre $\mathrm{PbO}$, les ions précurseurs sont $\mathrm{Ti}^{+}, \mathrm{Pb}^{+}$et des ions du type $\mathrm{MO}_{\mathrm{x}}^{+/-}($avec $\mathrm{x}=1,2$ ou 3 pour $\mathrm{M}=\mathrm{Ti}$ et $\mathrm{x}=1$ ou 2 pour $\mathrm{M}=\mathrm{Pb})$ ce qui conduit à la détection d'agrégats polycationiques de haute masse avec $\mathbf{n}=1$ à 17 [14]. Il a été également montré qu'en présence d'oxygène, ces agrégats polycationiques de haute masse étaient présents dans toute la plume d'ablation alors qu'ils sont détectés dans le plasma uniquement à partir d'une dizaine de millimètres du point d'impact sur la cible lorsque l'ablation est conduite sous vide [15]. Ces agrégats ont un rôle vis à vis du transport de l'oxygène : les agrégats du titane étant plus abondants que ceux à base de plomb, le transfert de l'oxygène depuis la cible va être essentiellement assuré par les espèces à base de titane, alors que le plomb qui est moins aisément fixé par l'oxygène rend le transfert de stœchiométrie difficile à assurer. Ce phénomène ajouté à la grande volatilité du plomb au contact du substrat porté à haute température induit un déficit intrinsèque en plomb dans les films de pérovskite obtenus par PLD. L'oxygène introduit sous pression contrôlée permet de mieux stabiliser les espèces oxygénées à base de plomb et de minimiser ainsi la perte du plomb lors du transfert. 


\section{CONCLUSION}

La prévision des conditions expérimentales employées en PLD pour obtenir la phase cristalline souhaitée avec la bonne composition chimique est en grande partie liée à la maîtrise des deux paramètres "pression d'oxygène" et "température du substrat". Ils doivent être adaptés en fonction du domaine de stabilité thermodynamique de la phase recherchée. L'oxygène moléculaire introduit dans l'enceinte conditionne non seulement le transfert de stœechiométrie de cet élément par des processus d'oxydation statique mais aussi la stabilisation des espèces oxygénées dans le plasma suite à des réactions de collision en phase gazeuse. Ce dernier point est d'importance vis à vis du transfert de stœechiométrie et de la fixation sur la couche mince des éléments volatils $(\mathrm{Pb}, \mathrm{Li}, \mathrm{Te}$..). Les processus de transfert dynamique provenant des réactions dans le plasma entre le gaz ambiant et les espèces ablatées de la cible est possible car l'énergie de dissociation de la molécule de dioxygène n'est que de $5,2 \mathrm{eV}$. En revanche, pour les films de nitrures synthétisés par PLD, la forte énergie de dissociation de l'azote moléculaire $\mathrm{N}_{2}(9,8 \mathrm{eV})$ rend plus difficile l'incorporation de l'azote dans les couches minces. Aussi, les conditions expérimentales utilisées pour l'ablation des oxydes ne peuvent pas être extrapolées au cas des nitrures pour lesquels le transfert de la stœchiométrie en azote entre la cible et le substrat nécessite l'assistance d'une source d'ions énergétiques.

\section{Remerciements}

L'auteur remercie N. Chaoui et J.F. Muller (LSMCL - Metz) pour leur contribution aux travaux de synthèse de films minces de la pérovskite $\mathrm{PbTiO}_{3}$ par $\mathrm{PLD}$ et aux études de formation des agrégats ionisés par spectrométrie de masse.

\section{Références}

1. E. Fogarassy, C. Fuchs, J.P. Stoquert, P. Siffert, J. Perrière, F. Rochet, J. Less Common Metals, 151 (1989), 249-253.

2. J.S. Horwitz, K.S. Grabowski, D.B. Chrysey, R.E. Leuchtner, Appl. Phys. Lett., 59 (1991), 1565 1568.

3. H. Tanaka, H. Tabata, T. Kawai, S. Kawai, Jpn, J. Appl. Phys., 33 (1994), 3b part 2, L451-L454

4. M. Morcrette, A. Gutierrez-Llorente, W. Seiler, J. Perrière, A. Laurent, P. Barboux, J. Appl. Phys. $88(2000), 5100-5106$.

5. M.R. Predtechensky, A.N. Smal, B.A. Kolesov, V.P. Ivanov, Appl. Supercond., 1 (1993), 10-12, 2005-2010

6. R. Gomez San Roman, R. Perez Cazero, C. Maréchal, J.P. Enard, J. Perrière, J. Appl. Phys., 80 (1996), 1787-1793

7. N. Chaoui, E. Millon, J.F. Muller, P. Ecker, W. Bieck, H.N. Migeon, Mat. Chem. Phys., 59 (1999), 114-119

8. R. Perez Cazero, R. Gomez San Roman, C. Maréchal, J.P. Enard, J. Perrière, Appl. Surf. Sci., 96-98 (1996), 697-702

9. J.S. Horwitz, K.S. Grabowski, D.B. Chrisey, R.E. Leuchtner, Appl. Phys. Lett., 59 (1991), 15651567

10.N.R. Barnes, R. Dat, D.J. Lichtenwaller, A.F. Schreiner, O. Auciello, O.E. Hankins, Chem. Mater., 7 (1995), 477-485

11.A. Mele, D. Consalvo, D. Strange, A. Giardini-Guidoni, R. Teghil, Int. J. Mass Spectrom. Ion Processes, 95 (1990), 359-373

12.X.Y. Chen, Z.C. Wu, Z.G. Liu, X.Y. Lei, Z.S. Sha, Appl. Phys. A, A6 (1998), 331-334

13.J.K. Gibson, J. Appl. Phys., 78 (1995), 1274-1280

14.N. Chaoui, E. Millon, J.F. Muller, Chem. Mater., 10 (1998), 3888-3894

15.S. Amuroso, V. Berardi, A. Dente, N. Spinelli, M. Armenante, R. Velotta, F. Fuso, M. Allegrini, E. Arimondo, J. Appl. Phys., 78 (1995), 494-504 